\title{
Ambiguous Representations of Semilattices, Imperfect Information, and Predicate Transformers
}

\author{
Oleh Nykyforchyn $^{1}$ (D) . Oksana Mykytsey ${ }^{2}$ (D)
}

Received: 6 May 2019 / Accepted: 13 September 2019 / Published online: 7 November 2019

(C) The Author(s) 2019

\begin{abstract}
Crisp and lattice-valued ambiguous representations of one continuous semilattice in another one are introduced and operation of taking pseudo-inverse of the above relations is defined. It is shown that continuous semilattices and their ambiguous representations, for which taking pseudo-inverse is involutive, form categories. Self-dualities and contravariant equivalences for these categories are obtained. Possible interpretations and applications to processing of imperfect information are discussed.
\end{abstract}

Keywords Ambiguous representation - Continuous semilattice - Duality of categories · Imperfect information

\section{Introduction}

The goal of this work is to generalize notions of crisp and $L$-fuzzy ambiguous representations introduced in [15] for closed sets in compact Hausdorff spaces. Fuzziness and roughness were combined to express the main idea that a set in one space can be represented with a set in another space, e.g., a 2D photo can represent 3D object. This representation is not necessarily unique, and the object cannot be recovered uniquely, hence we say "ambiguous representation".

It turned out that most of results of [15] can be extended to wider settings, namely to continuous semilattices, which are standard tool to represent partial information in denotational semantics of programming languages. Consider a computational process or a system which can be in different states, and these states can change, e.g., a game, position in which changes after moves of players. Assume that any party involved (e.g., a player) at a moment

Oleh Nykyforchyn

oleh.nyk@gmail.com

Oksana Mykytsey

oksana39@i.ua

1 Institute of Mathematics, Kasimir the Great University in Bydgoszcz, 2 Powstańców Wielkopolskich St., Bydgoszcz, 85090, Poland

2 Department of Mathematics and Computer Science, Vasyl' Stefanyk Precarpathian National University, 57 Shevchenka St., Ivano-Frankivsk, 76025, Ukraine 
of time can obtain only imperfect information about the state of the system ("imperfect" means that this information only reduces uncertainty but not necessarily eliminates it). All possible portions of information we can obtain in an observation form a domain of computation $S$ [3], which is usually required to be a continuous meet semilattice with zero (cf. [17] for a detailed explanation why continuous, semilattices are an appropriate tool for this purpose, and [5,9] for more information on continuous posets). If $x \leqslant y$ in $S$, then an information (a statement) $x$ is more specific (restrictive) than $y$. Respectively $0 \in S$ means "no information". The meet of $x$ and $y$ is the most specific piece of information including both $x$ and $y$ (as particular cases). It is not necessarily equivalent to " $x$ or $y$ " in the usual logical sense.

Example 1 Let $S$ be the set of all non-empty segments $[a, b] \subset[0,1]$ ordered by reverse inclusion. We regard $[a, b]$ as the statement "only points of $[a, b]$ have been selected". Then $\left[0, \frac{1}{3}\right] \wedge\left[\frac{2}{3}, 1\right]=[0,1]$, which is wider than " $\left[0, \frac{1}{3}\right]$ or $\left[\frac{2}{3}, 1\right]$ ".

Example 2 Let $S$ be the hyperspace $\exp X$ of all non-empty closed sets of a compactum $X$. Points of $X$ are possible states of the system, and $A \underset{\mathrm{cl}}{\subset} X$ represents the fact that one of the states $x \in A$ is achieved. Then $\exp X$ is ordered by reverse inclusion, hence $X \in$ $\exp X$ is the least element that means "anything can happen". The meet of $A$ and $B$ is their union, therefore can be interpreted as " $A$ or $B$ ". The ambiguous representations were first introduced and their properties proved for this particular case in [15].

Domain of computation is the primary interpretation of a continuous semilattice in this paper. We use monotonic binary- or lattice-valued predicates [8] on semilattices to express degrees of belief that certain pieces of information describe actual state of a system or of a process.

The goal of this work is to study relations between imperfect information on the same system or process obtained in different observation, e.g., how information changes after a step of a computational process or after a move in the game. We consider the introduced ambiguous representations to be an adequate tool for this task.

The paper is organized as follows. First necessary definitions and facts are given on continuous (semi-)lattices and monotone predicates. Then we define compatibilities, which are functions with values 0 and 1 that show whether two pieces of information can be valid together. Next ambiguous representations are introduced as crisp and $L$-fuzzy relations between continuous semilattices. Operation of taking pseudo-inverse is defined for these relations, its properties are proved, and classes of pseudo-invertible representations are investigated. It is shown that continuous semilattices and their pseudo-invertible crisp and $L$-fuzzy ambiguous representations form categories, and can be equivalently regarded as isotone mappings or linear operators between idempotent semimodules. Self-dualities and contravariant equivalences are constructed for these categories. We also discuss possible applications of the developed theory.

\section{Preliminaries}

We adopt the following definitions and notation, which are consistent with [5, 10]. Proofs of the facts below can also be found there. From now on, semilattice means meet semilattice, if otherwise is not specified. If a poset contains a bottom (a top) element, then it is denoted by 0 (resp. by 1). A top (a bottom) element in a semilattice is also called a unit (resp. a zero). 
For a partial order $\leqslant$ on a set $X$, the relation $\tilde{\leqslant}$, defined as $x \leqslant y \Longleftrightarrow y \leqslant x$, for $x, y \in X$, is a partial order called opposite to $\leqslant$, and $(X, \leqslant)^{o p}$ denotes the poset $(X, \tilde{\leqslant})$. If the original order $\leqslant$ is obvious, we write simply $X^{o p}$ for the reversed poset. We also apply ( ) to all notation to denote passing to the opposite order, i.e. write $\tilde{X}=X^{o p}$, sũp $=\inf , \tilde{0}=$ 1 etc. For a morphism $f:(X \leqslant) \rightarrow(Y, \leqslant)$ in a category $\mathcal{P}$ oset of posets and isotone (order preserving) mappings, let $f^{o p}$ be the same mapping, but regarded as $(X, \tilde{\leqslant}) \rightarrow(Y, \tilde{\leqslant})$. It is obvious that $f^{o p}$ is isotone as well, thus a functor $(-)^{o p}: \mathcal{P}$ oset $\rightarrow \mathcal{P}$ oset is obtained.

For a subset $A$ of a poset $(X, \leqslant)$, we denote

$$
A \uparrow=\{x \in X \mid a \leqslant x \text { for some } a \in A\}, A \downarrow=\{x \in X \mid x \leqslant a \text { for some } a \in A\} .
$$

If $A=A \uparrow(A=A \downarrow)$, then a set $A$ is called upper (resp. lower).

A topological meet (or join) semilattice is a semilattice $L$ carrying a topology such that the mapping $\wedge: L \times L \rightarrow L$ (resp. $\vee: L \times L \rightarrow L$ ) is continuous. A lattice $L$ with a topology such that both $\wedge: L \times L \rightarrow L$ and $\vee: L \times L \rightarrow L$ are continuous is called a topological lattice.

A poset $(X, \leqslant)$ is called complete if each non-empty subset $A \subset X$ has a least upper bound.

A set $A$ in a poset $(X, \leqslant)$ is directed (filtered) if, for all $x, y \in A$, there is $z \in A$ such that $x \leqslant z, y \leqslant z$ (resp. $z \leqslant x, z \leqslant y$ ). A poset is called directed complete (dcpo for short) if it has lowest upper bounds for all its directed subsets.

The Scott topology $\sigma(X)$ on $(X, \leqslant)$ consists of all those $U \subseteq X$ that satisfy $x \in$ $U \Leftrightarrow U \cap D \neq \varnothing$ for every $\leqslant-\operatorname{directed} D \subseteq X$ with a least upper bound $x$. Note that " $\Leftarrow "$ above implies $U=U \uparrow$.

In a dcpo $X$, a set is Scott closed iff it is lower and closed under suprema of directed subsets.

A mapping $f$ between dcpo's $X$ and $Y$ is Scott continuous, i.e. continuous w.r.t. $\sigma(X)$ and $\sigma(Y)$, if and only if it preserves suprema of directed sets.

Let $L$ be a poset. We say that $x$ is way below $y$ and write $x \ll y$ iff, for all directed subsets $D \subseteq L$ such that $\sup D$ exists, the relation $y \leq \sup D$ implies the existence of $d \in D$ such that $x \leq d$. "Way-below" relation is transitive and antisymmetric. An element satisfying $x \ll x$ is said to be compact or isolated from below, and in this case the set $\{x\} \uparrow$ is Scott open.

A poset $L$ is called continuous if, for each element $y \in L$, the set $y \Downarrow=\{x \in L \mid x \ll y\}$ is directed and its least upper bound is $y$. A domain is a continuous depo. If a domain is a semilattice, it is called a continuous semilattice.

A complete lattice $L$ is called completely distributive if, for each collection of sets $\left(M_{t}\right)_{t \in T}$ in $L$, the equality $\inf \left\{\sup M_{t} \mid t \in T\right\}=\sup \left\{\inf \left\{\alpha_{t} \mid t \in T\right\} \mid\left(\alpha_{t}\right)_{t \in T} \in \prod_{t \in T} M_{t}\right\}$ holds. This property implies distributivity, but the converse fails. Then both $L$ and $L^{o p}$ are continuous, and the join of Scott topologies on $L$ and $L^{o p}$ provides the unique compact Hausdorff topology on $L$ with a basis consisting of small sublattices (Lawson topology). In the sequel completely distributive lattices will be regarded with Lawson topologies.

For a subset $R \subset S_{1} \times S_{2} \times \cdots \times S_{n}$, an index $k \in\{1,2, \ldots, n\}$, and elements $\alpha_{1} \in S_{1}$, $\ldots, \alpha_{k-1} \in S_{k-1}, \alpha_{k+1} \in S_{k+1}, \ldots, \alpha_{n} \in S_{n}$, the set

$$
\begin{aligned}
& \underbrace{\operatorname{pr}_{1 \ldots(k-1)(k+1) \ldots n}}_{\text {all factors except } k \text {-th }}\left(R \cap\left(\left\{\alpha_{1}\right\} \times \cdots \times\left\{\alpha_{k-1}\right\} \times S_{k} \times\left\{\alpha_{k+1}\right\} \times \cdots \times\left\{\alpha_{n}\right\}\right)\right) \\
= & \left\{\alpha \in S_{k} \mid\left(\alpha_{1}, \ldots, \alpha_{k-1}, \alpha, \alpha_{k+1}, \ldots, \alpha_{n}\right) \in R\right\} \subset S_{k}
\end{aligned}
$$

is called a cut of $R$. We denote it $\alpha_{1} \ldots \alpha_{k-1} R \alpha_{k+1} \ldots \alpha_{n}$. 
The following obvious property is quite useful.

Lemma 1 Let $S_{1}, S_{2}, \ldots, S_{n}$ be dcpos, $R \subset S_{1} \times S_{2} \times \cdots \times S_{n}$. Then $R$ is Scott closed if and only if all its cuts are Scott closed.

Proof Necessity is obvious. To prove sufficiency, observe that all cuts of $R$ being Scott closed (hence lower sets) implies that $R$ is a lower set as well. Without loss of generality we can consider only the case $n=2$. Let a subset $D \subset R \subset S_{1} \times S_{2}$ be directed. Then the set $D^{\prime}=D \downarrow \subset R$ is directed as well and lower in $S_{1} \times S_{2}$, hence is the product $D_{1} \times D_{2}$ of directed lower sets $D_{1} \subset S_{1}$ and $D_{2} \subset S_{2}$. For any $x \in D_{1}$ the set $\{x\} \times D_{2}$ is contained in $R$, therefore $D_{2}$ is contained in the Scott closed cut $x R$. This implies that the least upper bound $b=\sup D_{2}$, which exists because $S_{2}$ is a dcpo, is an element of this cut, hence $(x, b) \in R$ for all $x \in D_{1}$. Thus $D_{1}$ is contained in the Scott closed cut $R b$, therefore $a=\sup D_{1}$ in the depo $S_{1}$ also belongs to this cut. We obtain that $(a, b)=\sup D^{\prime}=\sup D$ belongs to $R$ for any directed subset $D \subset R$, i.e., $R$ is Scott closed.

Similarly, for a subset $R \subset S_{1} \times S_{2} \times \cdots \times S_{n}$, an index $k \in\{1,2, \ldots, n\}$, and subsets $A_{1} \subset S_{1}, \ldots, A_{k-1} \subset S_{k-1}, A_{k+1} \subset S_{k+1}, \ldots, A_{n} \subset S_{n}$, we denote

$$
\begin{aligned}
& A_{1} \ldots A_{k-1} R A_{k+1} \ldots A_{n}=\left\{\alpha \in S_{k} \mid \text { there are } \alpha_{1} \in S_{1}, \ldots, \alpha_{k-1} \in S_{k-1},\right. \\
& \left.\alpha_{k+1} \in S_{k+1}, \ldots, \alpha_{n} \in S_{n} \text { such that }\left(\alpha_{1}, \ldots, \alpha_{k-1}, \alpha, \alpha_{k+1}, \ldots, \alpha_{n}\right) \in R\right\} .
\end{aligned}
$$

In the sequel $L$ will be a completely distributive lattice [5], which will be used to keep truth values ("degrees of belief"). Its top element 1 means "surely true", and the bottom element 0 means "quite impossible". In the simplest case $L=\mathbf{2}=\{0,1\}$, i.e., we obtain binary logic "yes/no".

In the sequel $D \rightarrow D^{\prime}$ denotes a Scott continuous mapping between domains $D$ and $D^{\prime}$, and $\left[D \rightarrow D^{\prime}\right]$ is the set of all such mappings. If $S$ and $S^{\prime}$ are continuous (meet) semilattices, then a Scott continuous meet preserving mapping is denoted with $S \rightarrow S^{\prime}$, with $\left[S \rightarrow S^{\prime}\right]$ being a notation for the respective sets of mappings. If the above posets have zero (bottom) elements, then $\left[D \rightarrow D^{\prime}\right]_{0}$ and $\left[S \rightarrow S^{\prime}\right]_{0}$ are the subsets consisting of the bottom-preserving mappings.

Let $\mathcal{S} \mathrm{em}_{\uparrow}$ be the category of all continuous semilattices and all Scott continuous mappings, and $\mathcal{S} \mathrm{em}_{0}$ be its subcategory consisting of all continuous semilattices with zeros and all Scott continuous zero-preserving semilattice morphisms.

Following [8], for a semilattice $S$ we call the elements of the set $\underline{M}_{[L]} S=\left[S \rightarrow L^{o p}\right]^{o p}$ $L$-fuzzy monotonic predicates on $S$. For $m \in\left[S \rightarrow L^{o p}\right]^{o p}$ and $a \in S$, we regard $m(a)$ as the truth value of $a$, hence it is required that $m(b) \leqslant m(a)$ for all $a \leqslant b$. The second ${ }^{o p}$ means that we order the fuzzy predicates pointwise, i.e. $m_{1} \leqslant m_{2}$ iff $m_{1}(a) \leqslant m_{2}(a)$ in $L$ (not in $L^{o p}$ !) for all $a \in S$. For $S$ with a least element 0 , consider also the subset $M_{[L]} S=$ $\left[S \rightarrow L^{o p}\right]_{0}^{o p} \subset \underline{M}_{[L]} S$ of all normalized predicates that take $0 \in S$ (no information) to $1 \in L$ (complete truth). It is natural to require $m(0)=1$ because we cannot be wrong if we say nothing. Observe that $M_{[L]} S$ is a complete sublattice of $\underline{M}_{[L]} S$.

In particular, a binary monotonic predicate $m \in \underline{M}_{[2]} S$ takes each piece $a$ of information to 1 if it is true or to 0 if it is wrong. Such predicate is completely determined with the Scott continuous set $m^{-1}(1) \subset S$ of true statements. On the other hand, each Scott closed set $F \subset S$ determines the binary monotonic predicate

$$
m_{F}(a)=\left\{\begin{array}{l}
1, a \in F, \\
0, a \notin F,
\end{array} \quad a \in S .\right.
$$


It follows from [4, Theorem 4] (although called "folklore knowledge" in [8]) that, for a domain $D$ and a completely distributive lattice $L$, the set $\left[D \rightarrow L^{o p}\right]$ is a completely distributive lattice, hence this is also valid for $\underline{M}_{[L]} S$, where $S$ is a continuous semilattice. If $S$ possesses a least element, then $M_{[L]} D$ is a completely distributive lattice as well. Infima in $\underline{M}_{[L]} S$ and $M_{[L]} S$ are calculated pointwise, but suprema need "adjustment"

$$
\left.\sup _{i} m_{i}\right)(a)=\inf \left\{\sup _{i} m_{i}\left(a^{\prime}\right) \mid a^{\prime} \ll a\right\}, \quad a \in S .
$$

\section{Compatibilities for Continuous Semilattices}

We use the results of [13] and denote by $S^{\wedge}$ the set of all (probably empty) Scott open filters in a continuous semilattice $S$ with zero except $S$ itself. We order $S^{\wedge}$ by inclusion, then $S^{\wedge}$ is a continuous semilattice with the bottom element $\varnothing$. Then $S^{\wedge}$ can be regarded as $[S \rightarrow\{0,1\}]_{0}$, i.e., its elements can be identified with the bottom-preserving meetpreserving Scott continuous maps $S \rightarrow\{0,1\}$ (the preimages of $\{1\}$ under such maps are precisely the non-trivial Scott open filters in $S$ ). For an arrow $f: S_{1} \rightarrow S_{2}$ in $\mathcal{S e m}_{0}$ the formula $f^{\wedge}(F)=f^{-1}(F), F \in S_{2}^{\wedge}$, determines the mapping $f^{\wedge}: S_{2}^{\wedge} \rightarrow S_{1}^{\wedge}$, which is an arrow in $\mathcal{S e m}_{0}$ as well, hence the contravariant functor $(-)^{\wedge}$ in $\mathcal{S e m}_{0}$ is obtained. The assignment $s \mapsto\left\{F \in S^{\wedge} \mid s \in F\right\}$ is an isomorphism $u_{S}: S \rightarrow S^{\wedge \wedge}$ which is a component of a natural transformation $u: \mathbf{1}_{\mathcal{S e m}_{0}} \rightarrow(-)^{\wedge \wedge}$, hence the functor $(-)^{\wedge}$ is involutive, i.e., is a self-duality. In fact it is a restriction of the Lawson duality [5].

We slightly change the terminology introduced in [13]:

Definition 1 Let $S, S^{\prime}$ be continuous semilattices with bottom elements respectively $0,0^{\prime}$. A mapping $P: S \times S^{\prime} \rightarrow\{0,1\}$ is called a compatibility if:

(1) $P$ is meet preserving in the both variables, and $P(0, y)=P\left(x, 0^{\prime}\right)=0$ for all $x \in S$, $y \in S^{\prime}$;

(2) $P$ is Scott continuous.

If, additionally, the following holds:

(3) $P$ separates elements of $S$ and of $S^{\prime}$, i.e.:

(3a) for each $x_{1}, x_{2} \in S$, if $P\left(x_{1}, y\right)=P\left(x_{2}, y\right)$ for all $y \in S^{\prime}$, then $x_{1}=x_{2}$;

(3b) for each $y_{1}, y_{2} \in S^{\prime}$, if $P\left(x, y_{1}\right)=P\left(x, y_{2}\right)$ for all $x \in S$, then $y_{1}=y_{2}$; then we call $P$ a separating compatibility.

The definition of (separating) compatibility is symmetric in the sense that the mapping $P^{\prime}: S^{\prime} \times S \rightarrow\{0,1\}, P^{\prime}(y, x)=P(x, y)$ is a (separating) compatibility as well, which we call the reverse compatibility. For compatibilities we use also infix notation $x P y \equiv$ $P(x, y)$.

We can consider a compatibility $P: S \times S^{\prime} \rightarrow\{0,1\}$ as a characteristic mapping of a binary relation $P \subset S \times S^{\prime}$, hence it is natural to denote $x P=\left\{y \in S^{\prime} \mid x P y=1\right\}$, $P y=\{x \in S \mid x P y=1\}$ for all $x \in S, y \in S^{\prime}$.

We interpret $P(x, y)=1$ as "pieces $x$ and $y$ of information are incompatible (cannot be valid simultaneously)", hence probably a longer term "incompatibility" would be more adequate. Meet-preservation in the first argument means that if $x_{1}$ and $x_{2}$ are incompatible (cannot be valid together) with $y$, then " $x_{1}$ or $x_{2}$ " is incompatible with $y$ as well, similarly for the second argument. A compatibility $P: S \times S^{\prime} \rightarrow\{0,1\}$ is separating if for all $x_{1} \neq x_{2}$ 
in $S$ there is $y \in S^{\prime}$ such that exactly one of $x_{i}$ is incompatible with $y$ w.r.t. $P$, similarly for $y_{1} \neq y_{2}$ in $S^{\prime}$ and $x \in S$. Then elements of $S^{\prime}$ can be regarded as "negative statements" about state of the system observed at $S$ : given $y \in S^{\prime}$ and a separating compatibility $P$, we declare impossible all $x \in S$ such that $(x, y) P 1$.

Example 3 Let $L$ be a completely distributive lattice, then so is $\tilde{L}=L^{o p}$. The mapping $P: L \times L^{o p} \rightarrow\{0,1\}$ defined as

$$
P(x, y)= \begin{cases}0, & y \ll x, \\ 1, & y \ll x,\end{cases}
$$

is a separating compatibility.

The following statement from [13] is of crucial importance:

Proposition 1 Let $S, S^{\prime}$ be continuous meet semilattices with bottom elements $0,0^{\prime}$ resp. If $P: S \times S^{\prime} \rightarrow\{0,1\}$ is a separating compatibility, then the mapping $i$ that takes each $x \in S$ to $x P$ is an isomorphism $S \rightarrow S^{\prime \wedge}$. Conversely, each isomorphism $i: S \rightarrow S^{\prime \wedge}$ is determined by the above formula for a unique separating compatibility $P: S \times S^{\prime} \rightarrow\{0,1\}$.

In particular, this together with the above example implies that $L^{\wedge} \cong L^{o p}$ for a completely distributive lattice $L$.

For a fixed separating compatibility $P: S \times S^{\prime} \rightarrow\{0,1\}$ and subsets $A \subset S, B \subset S^{\prime}$, the sets

$$
A^{\perp}=\left\{y \in S^{\prime} \mid x P y=0 \text { for all } x \in A\right\}, \quad B^{\perp}=\{x \in S \mid x P y=0 \text { for all } y \in B\}
$$

will be called the transversals of $A$ and $B$ respectively. In other words, $B^{\perp}$ is the set of all statements in $S$ that are compatible with all "negative statements" from $B \subset S^{\prime}$, similarly for $A^{\perp}$. Hence elements of $S^{\prime}$ prevent or prohibit elements of $S$, and vice versa. Separation means that each element is uniquely determined with all the elements it prohibits.

It is easy to see that $A^{\perp}$ and $B^{\perp}$ are Scott closed, and $A^{\perp \perp}=\left(A^{\perp}\right)^{\perp}$ is the Scott closure of $A \neq \varnothing$, i.e., the least Scott closed (hence lower) subset in $S$ that contains $A$, similarly for $B^{\perp \perp}$.

Obviously the transversal operation $(-)^{\perp}$ is antitone, i.e., $A \subset B$ implies $A^{\perp} \supset B^{\perp}$, and for a filtered family $\left\{A_{\alpha} \mid \alpha \in \mathcal{A}\right\}$ of closed lower sets the equality

$$
\left(\bigcap_{\alpha \in \mathcal{A}} A_{\alpha}\right)^{\perp}=\mathrm{Cl}\left(\bigcup_{\alpha \in \mathcal{A}} A_{\alpha}^{\perp}\right)
$$

is valid. This implies

$$
\bigcap_{\alpha \in \mathcal{A}} A_{\alpha}=\left(\mathrm{Cl}\left(\bigcup_{\alpha \in \mathcal{A}} A_{\alpha}^{\perp}\right)\right)^{\perp}=\left(\bigcup_{\alpha \in \mathcal{A}} A_{\alpha}^{\perp}\right)^{\perp} .
$$

\section{Category of Ambiguous Representations}

Definition 2 Let $S_{1}, S_{2}$ be continuous semilattices with zeros. An ambiguous representation of $S_{1}$ in $S_{2}$ is a binary relation $R \subset S_{1} \times S_{2}$ such that

(a) if $(x, y) \in R, x \leqslant x^{\prime}$ in $S_{1}$, and $y^{\prime} \leqslant y$ in $S_{2}$, then $\left(x^{\prime}, y^{\prime}\right) \in R$ as well; 
(b) for all $x \in S_{1}$ the set $x R=\left\{y \in S_{2} \mid(x, y) \in R\right\}$ is non-empty and closed under directed sups in $S_{2}$.

Observe that (a) implies $\left(x, 0_{2}\right) \in R$ for all $x \in S$. It also implies that $x R$ in (b) is a lower set, hence due to (b) is Scott closed.

Thus we can rearrange the requirements as follows, obtaining an equivalent definition:

Definition 3 For continuous semilattices $S_{1}, S_{2}$ with zeros, a binary relation $R \subset S_{1} \times S_{2}$ is an ambiguous representation of $S_{1}$ in $S_{2}$ if

$\left(\mathrm{a}^{\prime}\right)$ for all $x \in S_{1}$ the set $x R=\left\{y \in S_{2} \mid(x, y) \in R\right\}$ is non-empty and Scott closed (i.e., a directed complete lower set).

$\left(\mathrm{b}^{\prime}\right) \quad$ for all $y \in S_{2}$ the set $R y=\left\{x \in S_{1} \mid(x, y) \in R\right\}$ is an upper set.

We call such ambiguous representations crisp to distinguish them from $L$-fuzzy ambiguous representations that will be defined in the next section.

Recall that, for an ambiguous representation $R \subset S_{1} \times S_{2}$ and an element $x \in S_{1}$, the nonempty Scott closed set $x R \subset S_{2}$ determines the normalized binary monotonic predicate $m_{x R} \in M_{[2]} S_{2}$,

$$
m_{x R}(y)=\left\{\begin{array}{r}
1, \quad x R y, \\
0, \neg x R y,
\end{array} \quad y \in S_{2}\right.
$$

The correspondence $x \mapsto m_{x R}$, which we also denote with $R$, is isotone. Conversely, each isotone mapping $R: S_{1} \rightarrow M_{[2]} S_{2}$ uniquely determines an ambiguous representation $R \subset S_{1} \times S_{2}$ as follows: $x R y \Longleftrightarrow R(x)(y)=1$. Therefore in the sequel we can equivalently regard ambiguous representations either as relations or as isotone mappings. For each ambiguous representation $R$ of $S_{1}$ in $S_{2}$ we use notation $R: S_{1} \Rightarrow S_{2}$.

We interpret $x R y$ as "if $x$ is true, then $y$ can be (but not necessarily is) true" or " $y$ is attainable from $x$ ". Hence $x R$ is the set of all $y$ attainable from $x$ (under $R$ ), and $m_{x R}$ is the binary monotonic predicate that selects all elements of $S_{2}$ that can be true provided $x \in S_{1}$ is true.

Let separating compatibilities $P_{1}: S_{1} \times \hat{S}_{1} \rightarrow\{0,1\}, P_{2}=S_{2} \times \hat{S}_{2} \rightarrow\{0,1\}$ be fixed.

For an ambiguous representation $R: S_{1} \Rightarrow S_{2}$ define the relation $R^{\smile} \subset \hat{S}_{2} \times \hat{S}_{1}$ with the formula

$R^{\smile}=\left\{(\hat{y}, \hat{x}) \in \hat{S_{2}} \times \hat{S_{1}} \mid\right.$ if $x P_{1} \hat{x}=1$ for some $x \in S_{1}$, then there is $\left.y \in x R, y P_{2} \hat{y}=1\right\}$, or, equivalently

$R^{\smile}=\left\{(\hat{y}, \hat{x}) \in \hat{S_{2}} \times \hat{S}_{1} \mid\right.$ if $x \in S_{1}$ is such that $y P_{2} \hat{y}=0$ for all $y \in x R$, then $\left.x P_{1} \hat{x}=0\right\}$.

We explain why such definition has been chosen. If $x R y$ and $y P \hat{y}=1$, then $\hat{y}$ is incompatible with some "consequence" of $x$, hence $\hat{y}$ excludes $x$. If all the elements $x \in S_{1}$ incompatible with $\hat{x} \in \hat{S_{1}}$ are among those excluded by $\hat{y}$, then a "negative statement" $\hat{x}$ can be considered a "consequence" of a "negative statement" $\hat{y}$, which we denote $\hat{y} R \smile \hat{x}$.

It is easy to observe that $\hat{0}_{2} R^{\smile}=\left\{\hat{0}_{1}\right\}$, because $\hat{0}_{2} \in \hat{S}_{2}$ excludes nothing in $S_{1}$, therefore $\hat{0}_{1} \in \hat{S}_{1}$ is the only possible "consequence" of $\hat{0}_{2}$.

Obviously $R^{\smile}$ is an ambiguous representation $\hat{S}_{2} \Rightarrow \hat{S}_{1}$ as well, hence we can calculate $R^{\smile}=\left(R^{\smile}\right)^{\smile}: S_{1} \Rightarrow S_{2}$ using the reverse (in fact the same) separating compatibilities again. 
Proposition 2 For each ambiguous representation $R \subset S_{1} \times S_{2}$ the inclusion $R^{\approx} \subset R$ holds. The equality $R=R^{\smile}$ is equivalent to the conditions

(c) if $(x, y) \in R$ and $y^{\prime} \ll y$ in $S_{2}$, then there is $x^{\prime} \ll x$ in $S$ such that $\left(x^{\prime}, y^{\prime}\right) \in R$.

(d) if $\left(0_{1}, y\right) \in R$, then $y=0_{2}$.

Proof Necessity of (d) has been already explained. Assume it holds. Observe the validity of the formula

$$
\hat{y} R^{\smile}=\left\{x \in S_{1} \mid \hat{y} \in(x R)^{\perp}\right\}^{\perp} .
$$

Thus

$$
\bar{x} R^{\smile}=\left\{\hat{y} \in \hat{S_{2}} \mid \bar{x} \in\left(\hat{y} R^{\smile}\right)^{\perp}\right\}^{\perp}=\left\{\hat{y} \in \hat{S}_{2} \mid \bar{x} \in\left\{x \in S_{1} \mid \hat{y} \in(x R)^{\perp}\right\}^{\perp \perp}\right\}^{\perp} .
$$

Taking into account

$$
\bar{x} \in\left\{x \in S_{1} \mid \hat{y} \in(x R)^{\perp}\right\}^{\perp \perp} \Leftarrow \hat{y} \in(\bar{x} R)^{\perp},
$$

we obtain

$$
\bar{x} R^{\asymp} \subset\left\{\hat{y} \in \hat{S_{2}} \mid \hat{y} \in(\bar{x} R)^{\perp}\right\}^{\perp}=(\bar{x} R)^{\perp \perp}=\bar{x} R
$$

for all $\bar{x} \in S_{1}$, which is the required inclusion.

The set $\left\{x \in S_{1} \mid \hat{y} \in(x R)^{\perp}\right\}$ is lower, and by (d) is non-empty, therefore its double transversal is the closure, thus

$$
\begin{aligned}
\bar{x} R^{\Xi} & =\left\{\hat{y} \in \hat{S}_{2} \mid \bar{x} \in \operatorname{Cl}\left\{x \in S_{1} \mid \hat{y} \in(x R)^{\perp}\right\}\right\}^{\perp} \\
& =\left\{\hat{y} \in \hat{S}_{2} \mid \hat{y} \in(x R)^{\perp} \text { for all } x \ll \bar{x}\right\}^{\perp} \\
& =\left(\bigcap\left\{(x R)^{\perp} \mid x \ll \bar{x}\right\}\right)^{\perp} .
\end{aligned}
$$

The family $\left\{\{x R)^{\perp} \mid x \ll \bar{x}\right\}$ of closed lower sets is filtered, hence the transversal of its intersection equals

$$
\mathrm{Cl}\left(\bigcup\left\{(x R)^{\perp \perp} \mid x \ll \bar{x}\right\}\right)=\mathrm{Cl}(\bigcup\{x R \mid x \ll \bar{x}\}),
$$

and the equality $R^{\smile}=R$ is equivalent to

$$
\bar{x} R=\operatorname{Cl}(\bigcup\{x R \mid x \ll \bar{x}\}),
$$

which in fact is the condition (c).

The equality $R^{\smile}=R$ implies $\left(R^{\smile}\right)^{\smile}=R^{\smile}$, hence, if (c), (d) hold for $R$, then they hold for $R^{\smile}$ as well. Therefore on such ambiguous representations the operation ( $)^{\smile}$ is involutive, and we call each of $R$ and $R^{\smile}$ the pseudo-inverse to the other one. The ambiguous representations satisfying (c), (d) are called pseudo-invertible.

Proposition 3 An ambiguous representation $R \subset S_{1} \times S_{2}$ is pseudo-invertible if and only if, when regarded as a mapping $S_{1} \rightarrow M_{[2]} S_{2}$, it is Scott continuous and bottom-preserving.

Proof Clearly (d) is equivalent to $R\left(0_{1}\right)=m_{\left\{0_{2}\right\}}$, and $m_{\left\{0_{2}\right\}}$ is the least element of $M_{[2]} S_{2}$, thus (d) means that $R$ is bottom-preserving.

Let $R$ be pseudo-invertible, hence satisfy (c). To show Scott continuity of $R: S_{1} \rightarrow$ $M_{[2]} S_{2}$, it is sufficient to prove that $R\left(x_{0}\right)=\sup \left\{R(x) \mid x \ll x_{0}\right\}$ for all $x_{0} \in S_{1}$, which in fact means that $x_{0} R=\mathrm{Cl}\left(\bigcup_{x \ll x_{0}} x R\right)$. If $y_{0} \in x_{0} R$, then for all $y \ll y_{0}$ by (c) there is $x \ll$ 
$x_{0}$ such that $x R y$, hence $y \in \bigcup_{x \ll x_{0}} x R$. Taking into account $y_{0} \in \operatorname{Cl}\left\{y \in S_{2} \mid y \ll y_{0}\right\}$, we obtain $x_{0} R \subset \mathrm{Cl}\left(\bigcup_{x \ll x_{0}} x R\right)$. The reverse inclusion is immediate.

Now let $R: S_{1} \rightarrow M_{[2]} S_{2}$ be Scott continuous, and $x_{0} R y_{0}$. Then the limit of the directed net of binary monotonic predicates $m_{x}, x \ll x_{0}$, i.e., their lowest upper bound, is equal to $m_{x_{0} R}$, hence

$$
\inf \left\{\sup _{x \ll x_{0}} m_{x R}(y) \mid y \ll y_{0}\right\}=m_{x_{0} R}\left(y_{0}\right)=1,
$$

i.e., for all $y \ll y_{0}$ there is $x \ll x_{0}$ such that $m_{x R}(y)=1 \Longleftrightarrow x R y$. Therefore (c) is valid, and, if (d) is valid as well, then $R$ is pseudo-invertible.

One of the reasons to consider this subclass is that, if we compose ambiguous representations as relations, i.e., for $R \subset S_{1} \times S_{2}, Q \subset S_{2} \times S_{3}$ :

$$
R Q=\left\{(x, z) \in S_{1} \times S_{3} \mid \text { there is } y \in S_{2} \text { such that }(x, y) \in R,(y, z) \in Q\right\},
$$

then the resulting relation can fail to satisfy closedness in the condition (b) of the definition of ambiguous representation, hence ambiguous representations do not form a category.

To improve things, redefine the composition as

$$
\begin{aligned}
R ; Q & =\left\{(x, z) \in S_{1} \times S_{3} \mid z \in \mathrm{Cl}(x R Q)\right\} \\
& =\left\{(x, z) \in S_{1} \times S_{3} \mid \text { for all } z^{\prime} \ll z \text { there is } y \in S_{2} \text { such that }(x, y) \in R,\left(y, z^{\prime}\right) \in Q\right\} .
\end{aligned}
$$

Now closedness is at hand, but the composition ";" is not associative.

Lemma 2 For all ambiguous representations $R \subset S_{1} \times S_{2}, Q \subset S_{2} \times S_{3}$ the inclusion $Q^{\smile ;} R^{\smile} \subset(R ; Q)^{\smile}$ holds.

Proof Since $\hat{z} Q^{\smile ;} R^{\smile}=\mathrm{Cl}\left(z Q^{\smile} R^{\smile}\right)$ for all $\hat{z} \in \hat{S_{3}}$, and $\hat{z}(R ; Q)^{\smile}$ is closed in $S_{1}$, it is sufficient to prove $Q^{\smile} R^{\smile} \subset(R ; Q)^{\smile}$.

If $(\hat{z}, \hat{x}) \in Q^{\smile} R^{\smile}$, then choose $\hat{y} \in \hat{S_{2}}$ such that $(\hat{z}, \hat{y}) \in Q^{\smile}$ and $(\hat{y}, \hat{x}) \in R^{\smile}$, and combine

$$
\begin{aligned}
& \text { if } x P_{1} \hat{x}=1 \text { for some } x \in S_{1} \text {, then there is } y \in x R, \quad y P_{2} \hat{y}=1 \text {, } \\
& \text { if } y P_{2} \hat{y}=1 \text { for some } y \in S_{2} \text {, then there is } z \in y Q, z P_{3} \hat{z}=1
\end{aligned}
$$

to obtain

$$
\text { if } x P_{1} \hat{x}=1 \text { for some } x \in S_{1} \text {, then there is } z \in x R Q, z P_{3} \hat{z}=1 \text {. }
$$

Moreover the latter $z$ belongs to $x R ; Q$, hence $(\hat{z}, \hat{x}) \in(R ; Q)^{\smile}$.

Corollary 1 Let ambiguous representations $R \subset S_{1} \times S_{2}, Q \subset S_{2} \times S_{3}$ be pseudo-invertible. Then $Q^{\smile ;} R^{\smile}=(R ; Q)^{\smile}$, and the composition $R ; Q$ is pseudo-invertible as well.

Proof By the above and taking into account that $(-)^{\smile}$ is isotone:

$$
R ; Q=R^{\smile ;} Q^{\asymp} \subset\left(Q^{\smile ;} R^{\smile}\right)^{\smile} \subset(R ; Q)^{\asymp},
$$

and the reverse inclusion is known, hence $R ; Q=(R ; Q)^{\smile}=\left(Q^{\smile ;} R^{\smile}\right)^{\smile}$, i.e., $R ; Q$ is pseudo-invertible. Apply $(-)^{\smile}$ to this again and obtain $(R ; Q)^{\smile}=\left(Q^{\smile ;} R^{\smile}\right)^{\smile}=Q^{\smile ;} R^{\smile}$. 
Proposition 4 Composition “;” of the pseudo-invertible ambiguous representations is associative.

Proof Recall that, for ambiguous representations $R \subset S_{1} \times S_{2}, Q \subset S_{2} \times S_{3}$, the composition is calculated as

$R ; Q=\left\{(x, z) \in S_{1} \times S_{3} \mid\right.$ for all $z^{\prime} \ll z$ there is $y \in S_{2}$ such that $\left.(x, y) \in R,\left(y, z^{\prime}\right) \in Q\right\}$.

It is also important that, for elements $a \ll c$ in a continuous semilattice, there is an element $b$ such that $a \ll b \ll c$.

Now, let $(x, t) \in R ;(Q ; T)$. For any $t^{\prime} \ll t$ choose $t^{\prime \prime}$ such that $t^{\prime} \ll t^{\prime \prime} \ll t$, then there is $y \in S_{2}$ such that $(x, y) \in R,\left(y, t^{\prime \prime}\right) \in Q ; T$. The latter implies that there is $z \in S_{3}$ such that $(y, z) \in Q,\left(z, t^{\prime}\right) \in T$.

Similarly, let $(x, t) \in(R ; Q) ; T$, then for all $t^{\prime} \ll t$ choose $t^{\prime} \ll t^{\prime \prime} \ll t$, and there is $z^{\prime} \in S_{3}$ such that $\left(x, z^{\prime}\right) \in R ; Q,\left(z^{\prime}, t^{\prime \prime}\right) \in T$. Pseudo-invertibility of $T$ implies the existence of $z \ll z^{\prime}$ such that $\left(z, t^{\prime}\right) \in T$ as well. There is also $y \in S_{2},(x, y) \in R,(y, z) \in Q$.

On the other hand, if, for $x \in S_{1}, t \in S_{4}$, elements $y \in S_{2}, z \in S_{3}$ exist for all $t^{\prime} \ll t$ such that $(x, y) \in R,(y, z) \in Q,\left(z, t^{\prime}\right) \in T$, then $\left(x, t^{\prime}\right) \in R Q T$, hence $\left(x, t^{\prime}\right) \in R(Q ; T)$ and $\left(x, t^{\prime}\right) \in(R ; Q) T$, which in turn implies both $(x, t) \in R ;(Q ; T)$ and $(x, t) \in(R ; Q) ; T$. Thus $R ;(Q ; T)=(R ; Q) ; T$.

It is easy to verify that, for a continuous semilattice $S$ with zero, the relation $E_{S}=$ $\{(x, y) \in S \times S \mid y \leqslant x\}$ is a pseudo-invertible ambiguous representation that is a neutral element for composition. Thus:

Proposition 5 All continuous semilattices with bottom elements and all pseudo-invertible ambiguous representation form a category $\mathcal{S} \mathrm{em} \mathcal{P} \mathcal{R}$, which contain $\mathcal{S e m}_{0}$ as a subcategory.

An obvious embedding $\mathcal{S e m}_{0} \rightarrow \mathcal{S e m} \mathcal{P} \mathcal{R}$ is of the form: $I S=S$ for an object $S$, and If $=\left\{(x, y) \in S_{1} \times S_{2} \mid y \leqslant f(x)\right\}$ for an arrow $f: S_{1} \rightarrow S_{2}$.

We denote an arrow $R$ from $S_{1}$ to $S_{2}$ in $\mathcal{S}$ em $\mathcal{P} \mathcal{R}$ with $R: S_{1} \Rightarrow S_{2}$ and use for the composition of $R$ and $Q$ the synonymic notations $R$; $Q$ (in direct order) and $Q \circ R$ (in reverse order) both in $\mathcal{S}$ em 0 and $\mathcal{S}$ em $\mathcal{P} \mathcal{R}$.

Proposition 6 The correspondence $(-)^{\smile}$ is an involutive contravariant functor (a selfduality) in $\mathcal{S e m} \mathcal{P} \mathcal{R}$, which is an extension of the functor $(-)^{\wedge}$ in $\mathcal{S e m}_{0}$.

\section{Category of L-Fuzzy Ambiguous Representations}

Now we extend the notion of ambiguous representation to lattice-valued relations in the spirit of [12] and [13].

Definition 4 Let $S_{1}, S_{2}$ be continuous semilattices with zeros $0_{1}$ and $0_{2}$ resp., $L$ a completely distributive lattice with a bottom element 0 and a top element 1 . An $L$-fuzzy ambiguous representation (or an L-ambiguous representation for short) of $S_{1}$ in $S_{2}$ is a ternary relation $R \subset S_{1} \times S_{2} \times L$ such that

(a) if $(x, y, \alpha) \in R, x \leqslant x^{\prime}$ in $S_{1}, y^{\prime} \leqslant y$ in $S_{2}$, and $\alpha^{\prime} \leqslant \alpha$ in $L$, then $\left(x^{\prime}, y^{\prime}, \alpha^{\prime}\right) \in R$ as well; 
(b) for all $x \in S_{1}$ the set $x R=\left\{(y, \alpha) \in S_{2} \times L \mid(x, y, \alpha) \in R\right\}$ is Scott closed in $S_{2} \times L$ and contains all the elements of the forms $\left(0_{2}, \alpha\right)$ and $(y, 0)$;

(c) for all $x \in S_{1}, y \in S_{2}, \alpha, \beta \in L$, if $(x, y, \alpha) \in R,(x, y, \beta) \in R$, then $(x, y, \alpha \vee \beta) \in R$.

The following definition is equivalent.

Definition 5 For continuous semilattices $S_{1}, S_{2}$ with zeros and a completely distributive lattice $L$, a ternary relation $R \subset S_{1} \times S_{2} \times L$ is an L-ambiguous representation of $S_{1}$ in $S_{2}$ if

$\left(\mathrm{a}^{\prime}\right)$ for all $y \in S_{2}, \alpha \in L$ the set $R y \alpha=\left\{x \in S_{1} \mid(x, y, \alpha) \in R\right\}$ is an upper set in $S_{1}$;

$\left(\mathrm{b}^{\prime}\right)$ for all $x \in S_{1}, \alpha \in L$ the set $x R \alpha=\left\{y \in S_{2} \mid(x, y, \alpha) \in R\right\}$ is non-empty and Scott closed in $S_{2}$;

(c') for all $x \in S_{1}, y \in S_{2}$ the set $x y R=\{\alpha \in L \mid(x, y, \alpha) \in R\}$ is non-empty, directed, and Scott closed in $L$.

Obviously, due to complete distributivity of $L,\left(\mathrm{c}^{\prime}\right)$ is equivalent to any of the following properties:

$\left(\mathrm{c}^{\prime \prime}\right)$ for all $x \in S_{1}, y \in S_{2}$ the set $x y R=\{\alpha \in L \mid(x, y, \alpha) \in R\}$ is a non-empty directed lower set such that, if $\beta \in x y R$ for all $\beta \ll \alpha$, then $\alpha \in x y R$; or

( $\left.\mathrm{c}^{\prime \prime \prime}\right)$ for all $x \in S_{1}, y \in S_{2}$ the set $x y R$ is a lower set with a greatest element (i.e., a set of the form $\{\alpha\} \downarrow)$.

Fix $x \in S_{1}$, then due to ( $\left.\mathrm{c}^{\prime \prime \prime}\right)$ the formula

$$
m_{x R}(y)=\max \{\alpha \in L \mid(x, y, \alpha) \in R\}, \quad y \in S_{2},
$$

defines a normalized $L$-fuzzy monotonic predicate $m_{x R} \in M_{[L]} S_{2}$, and the correspondence $R$ that takes each $x$ to $m_{x R}$ is an isotone mapping $S_{1} \rightarrow M_{[L]} S_{2}$. On the other hand, similarly to the binary case, each isotone mapping $R: S_{1} \rightarrow M_{[L]} S_{2}$ determines the $L$-ambiguous representation

$$
R=\left\{(x, y, \alpha) \in S_{1} \times S_{2} \times L \mid \alpha \leqslant R(x)(y)\right\},
$$

and these conversions are mutually inverse. Hence we can equivalently regard $L$-ambiguous representations either as relations or as isotone mappings, whatever is more convenient.

An $L$-ambiguous representation of $S_{1}$ in $S_{2}$ is denoted with $R: S_{1} \Rightarrow^{L} S_{2}$. In this paper we interpret $(x, y, \alpha)$ as "if $x$ is true, then the truth value of $y$ is at least $\alpha$ " or "given $x, y$ is attainable with degree of belief at least $\alpha$ ". By definition, for all $x$ and $y$ there is the greatest such $\alpha$, which can also be treated as quality/precision of representation of one piece of information with another one.

Observe also that $\left(\mathrm{a}^{\prime}\right)+\left(\mathrm{b}^{\prime}\right)$ mean that, for all $\alpha \in L$, the cut $R \alpha=\left\{(x, y) \in S_{1} \times S_{2} \mid\right.$ $(x, y, \alpha) \in R\}$ is a (crisp) ambiguous representation of $S_{1}$ in $S_{2}$ as defined in the previous section. We will call it the $\alpha$-cut of $R$ and denote $R_{\alpha}$.

We assume again that separating compatibilities $P_{1}: S_{1} \times \hat{S}_{1} \rightarrow\{0,1\}, P_{2}=S_{2} \times \hat{S}_{2} \rightarrow$ $\{0,1\}$ are fixed.

For an ambiguous representation $R \subset S_{1} \times S_{2} \times L$ define the relation $R^{\smile} \subset \hat{S}_{2} \times \hat{S}_{1} \times L$ through its $\alpha$-cuts as follows:

$$
\left(R^{\smile}\right)_{\alpha}= \begin{cases}\bigcap_{\beta \ll \alpha}\left(R_{\beta}\right)^{\smile}, & \alpha \neq 0, \\ \hat{S}_{2} \times \hat{S}_{1}, & \alpha=0,\end{cases}
$$


or, equivalently, with the formulae

$$
\begin{aligned}
& R^{\smile}=\left\{(\hat{y}, \hat{x}, \alpha) \in \hat{S_{2}} \times \hat{S_{1}} \times L \mid \text { if } \beta \ll \alpha \neq 0 \text { and } x P_{1} \hat{x}=1 \text { for some } x \in S_{1},\right. \\
&\text { then there is } \left.(y, \beta) \in x R, y P_{2} \hat{y}=1\right\},
\end{aligned}
$$

or

$$
\begin{gathered}
R^{\smile}=\left\{(\hat{y}, \hat{x}) \in \hat{S}_{2} \times \hat{S}_{1} \times L \mid \text { if } \beta \ll \alpha \neq 0 \text { and } x \in S_{1} \text { is such that } y P_{2} \hat{y}=0\right. \\
\left.\quad \text { for all }(y, \beta) \in x R \text {, then } x P_{1} \hat{x}=0\right\} .
\end{gathered}
$$

A shorter formula uses transversals:

$$
\hat{y}\left(R^{\smile}\right)_{\alpha}= \begin{cases}\bigcap_{\beta \ll \alpha}\left\{x \in S_{1} \mid \hat{y} \in\left(x R_{\beta}\right)^{\perp}\right\}^{\perp}, & \alpha \neq 0, \\ \hat{S}_{1}, & \alpha=0 .\end{cases}
$$

Proposition 7 The relation $R^{\smile}$ is an L-ambiguous representation as well.

Proof For the intersection of crisp ambiguous representations is a crisp ambiguous representation as well, $\left(\mathrm{a}^{\prime}\right)+\left(\mathrm{b}^{\prime}\right)$ for $R^{\smile}$ are immediate. To verify $\mathrm{c}^{\prime \prime}$, assume that $\beta \in \hat{y} \hat{x} R^{\smile}$ for all $\beta \ll \alpha \neq 0$, i.e., $(\hat{y}, \hat{x}) \in \bigcap_{\beta \ll \alpha} \bigcap_{\gamma \ll \beta}\left(R_{\gamma}\right)^{\smile}$. In a completely distributive lattice $L$ we have $\gamma \ll \alpha$ if and only if there is $\beta$ such that $\gamma \ll \beta \ll \alpha$, hence $(\hat{y}, \hat{x}) \in \bigcap_{\gamma \ll \alpha}\left(R_{\gamma}\right)^{\smile}$, which implies $\alpha \in \hat{y} \hat{x} R^{\smile}$.

Obviously $\hat{y} \hat{x} R^{\smile}$ is a lower set that contains 0 . Show that it is directed. If $\alpha, \beta \in \hat{y} \hat{x} R^{\smile \text {, }}$ then for all $\gamma \ll \alpha \vee \beta$ there are $\alpha^{\prime} \ll \alpha, \beta^{\prime} \ll \beta$ such that $\gamma \leqslant \alpha^{\prime} \vee \beta^{\prime}$. Therefore

$$
\begin{aligned}
\hat{x} & \in \hat{y}\left(R_{\alpha^{\prime}}\right)^{\smile} \cap \hat{y}\left(R_{\beta^{\prime}}\right)^{\smile} \\
& =\left\{x \in S_{1} \mid \hat{y} \in\left(x R_{\alpha^{\prime}}\right)^{\perp}\right\}^{\perp} \cap\left\{x \in S_{1} \mid \hat{y} \in\left(x R_{\beta^{\prime}}\right)^{\perp}\right\}^{\perp} \\
& =\left\{x \in S_{1} \mid \hat{y} \in\left(x R_{\alpha^{\prime}}\right)^{\perp} \text { or } \hat{y} \in\left(x R_{\beta^{\prime}}\right)^{\perp}\right\}^{\perp} \\
& \subset\left\{x \in S_{1} \mid \hat{y} \in\left(x R_{\alpha^{\prime} \vee \beta^{\prime}}\right)^{\perp}\right\}^{\perp}=\hat{y}\left(R_{\alpha^{\prime} \vee \beta^{\prime}}\right)^{\smile} \subset \hat{y}\left(R_{\gamma}\right)^{\smile .}
\end{aligned}
$$

Thus $\alpha \vee \beta \in \hat{y} \hat{x} R^{\smile}$, and the latter set is directed in $L$.

Hence $R^{\smile}=\left(R^{\smile}\right)^{\smile} \subset S_{1} \times S_{2} \times L$ is an $L$-ambiguous representation as well.

Proposition 8 For each ambiguous representation $R \subset S_{1} \times S_{2} \times$ L the inclusion $R^{\smile} \subset R$ holds. The equality $R=R \approx$ is equivalent to the conditions

(d) if $(x, y, \alpha) \in R, y^{\prime} \ll y$ in $S_{2}$, and $\alpha^{\prime} \ll \alpha$ in L, then there is $x^{\prime} \ll x$ in $S$ such that $\left(x^{\prime}, y^{\prime}, \alpha^{\prime}\right) \in R$.

(e) if $\left(0_{1}, y, \alpha\right) \in R, \alpha \neq 0$, then $y=0_{2}$.

Proof Necessity of (e) is an immediate corollary of the analogous property (d) for the pseudo-inverses of crisp ambiguous representations. Assume that (e) holds. Recall that

$$
\hat{y}\left(R^{\smile}\right)_{\beta}=\bigcap_{\gamma \ll \beta}\left\{x \in S_{1} \mid \hat{y} \in\left(x R_{\gamma}\right)^{\perp}\right\}^{\perp}=\left(\bigcup_{\gamma \ll \beta}\left\{x \in S_{1} \mid \hat{y} \in\left(x R_{\gamma}\right)^{\perp}\right\}\right)^{\perp} .
$$


Hence

$$
\begin{aligned}
x\left(R^{\smile}\right)_{\alpha} & =\bigcap_{\beta \ll \alpha}\left\{\hat{y} \in \hat{S}_{2} \mid x \in\left(\hat{y}\left(R^{\smile}\right)_{\beta}\right)^{\perp}\right\}^{\perp} \\
& =\bigcap_{\beta \ll \alpha}\left\{\hat{y} \in \hat{S}_{2} \mid x \in\left(\bigcup_{\gamma \ll \beta}\left\{x^{\prime} \in S_{1} \mid \hat{y} \in\left(x^{\prime} R_{\gamma}\right)^{\perp}\right\}\right)^{\perp \perp}\right\}^{\perp}
\end{aligned}
$$

(double transversal of the non-empty by (e) set is its Scott closure)

$=\bigcap_{\beta \ll \alpha}\left\{\hat{y} \in \hat{S}_{2} \mid x \in \mathrm{Cl}\left(\bigcup_{\gamma \ll \beta}\left\{x^{\prime} \in S_{1} \mid \hat{y} \in\left(x^{\prime} R_{\gamma}\right)^{\perp}\right\}\right)\right\}^{\perp}$

(Scott closure of a lower set $A$ consists of all points approximated by elements of $A$ )

$$
\begin{aligned}
& =\bigcap_{\beta \ll \alpha}\left\{\hat{y} \in \hat{S}_{2} \mid \text { for all } x^{\prime} \ll x \text { there is } \gamma \ll \beta \text { such that } \hat{y} \in\left(x^{\prime} R_{\gamma}\right)^{\perp}\right\}^{\perp} \\
& \stackrel{*}{=} \bigcap_{\beta \ll \alpha}\left\{\hat{y} \in \hat{S}_{2} \mid \text { for all } x^{\prime} \ll x \quad \hat{y} \in\left(x^{\prime} R_{\beta}\right)^{\perp}\right\}^{\perp} \\
& =\bigcap_{\beta \ll \alpha}\left(\{x\} \Downarrow R_{\beta}\right)^{\perp \perp} \subset \bigcap_{\beta \ll \alpha}\left(x R_{\beta}\right)^{\perp \perp}=\bigcap_{\beta \ll \alpha}\left(x R_{\beta}\right)=x R_{\alpha},
\end{aligned}
$$

therefore $R^{\approx} \subset R$. Clarify why the " $=$ " sign with an asterisk is valid. Obviously, if $\gamma \ll \beta$ and $\hat{y} \in\left(x^{\prime} R_{\gamma}\right)^{\perp}$, then $\hat{y} \in\left(x^{\prime} R_{\beta}\right)^{\perp}$, and $(-)^{\perp}$ is antitone, hence " $\supset$ " is immediate. On the other hand,

there is $\gamma \ll \beta$ such that for all $x^{\prime} \ll x \hat{y} \in\left(x^{\prime} R_{\gamma}\right)^{\perp}$ $\Longrightarrow$ for all $x^{\prime} \ll x$ there is $\gamma \ll \beta$ such that $\hat{y} \in\left(x^{\prime} R_{\gamma}\right)^{\perp}$,

hence

$$
\begin{aligned}
& \bigcap_{\beta \ll \alpha}\left\{\hat{y} \in \hat{S}_{2} \mid \text { for all } x^{\prime} \ll x \text { there is } \gamma \ll \beta \text { such that } \hat{y} \in\left(x^{\prime} R_{\gamma}\right)^{\perp}\right\}^{\perp} \\
\subset & \bigcap_{\beta \ll \alpha}\left\{\hat{y} \in \hat{S}_{2} \mid \text { there is } \gamma \ll \beta \text { such that for all } x^{\prime} \ll x \hat{y} \in\left(x^{\prime} R_{\gamma}\right)^{\perp}\right\}^{\perp} \\
= & \bigcap_{\gamma \ll \beta \ll \alpha}\left\{\hat{y} \in \hat{S}_{2} \mid \text { for all } x^{\prime} \ll x \hat{y} \in\left(x^{\prime} R_{\gamma}\right)^{\perp}\right\}^{\perp} \\
= & \bigcap_{\gamma \ll \alpha}\left\{\hat{y} \in \hat{S}_{2} \mid \text { for all } x^{\prime} \ll x \hat{y} \in\left(x^{\prime} R_{\gamma}\right)^{\perp}\right\}^{\perp},
\end{aligned}
$$

therefore " $\subset$ " (after renaming $\gamma$ with $\beta$ ) is also obtained.

It is also clear that, for $R^{\Xi}=R$ to be valid, it is necessary and sufficient that the only " $C$ " in the above sequence is "=", i.e., each $y \in x R_{\alpha}$ must be a closure point of all lower sets $\{x\} \Downarrow R_{\beta}$ for $\beta \ll \alpha$, which in fact is (d). 
The $L$-ambiguous representations $R$ that satisfy $R=R^{\smile}$ are also called pseudoinvertible. Similarly to the binary case:

Proposition 9 An L-ambiguous representation $R \subset S_{1} \times S_{2} \subset L$ is pseudo-invertible if and only if, when regarded as a mapping $S_{1} \rightarrow M_{[L]} S_{2}$, it is Scott continuous and bottompreserving.

Proof is also obtained mutatis mutandis.

To define composition of $L$-ambiguous representations, we need an additional operation $*: L \times L \rightarrow L$ that makes $L=(L, *)$ a unital quantale, i.e., this operation is infinitely distributive w.r.t. supremum in both variables (hence Scott continuous) and 1 is a two sided unit for " $*$ ". Note that commutativity is not demanded, hence from now on " $*$ " is a (possibly) noncommutative lower semicontinuous conjunction for an $L$-valued fuzzy logic [2, 7]. The operation $\alpha \hat{*} \beta \equiv \beta * \alpha$ satisfies the same requirements, hence $\hat{L}=(L, \hat{*})$ is a unital quantale as well.

Then, for $L$-ambiguous representations $R \subset S_{1} \times S_{2} \times L$ and $Q \subset S_{2} \times S_{3} \times L$, the composition $R * Q$ can be defined in a manner usual for $L$-fuzzy relations:

$$
\begin{aligned}
R * Q=\left\{(x, z, \alpha) \in S_{1} \times S_{3} \times L \mid\right. & \\
& \left.\alpha \leqslant \sup \left\{\beta * \gamma \mid \text { there is } y \in S_{3} \text { such that }(x, y, \beta) \in R,(y, z, \gamma) \in Q\right\}\right\} .
\end{aligned}
$$

Similarly to the case of crisp ambiguous representations, this composition is associative, but $R * Q$ can fail to be an $L$-ambiguous representation, namely (b) is not always valid. Therefore we "improve" the composition as follows: $R^{*} Q \subset S_{1} \times S_{3} \times L$ is such that $x R * Q=\mathrm{Cl}(x R * Q)$ for all $x \in S_{1}$. Here is an expanded version of the latter definition: for $x \in S_{1}, z \in S_{3}, \alpha \in L$ we have $(x, z, \alpha) \in R^{*}, Q$ if and only if for all $z^{\prime} \ll z, \alpha^{\prime} \ll \alpha$ there are $n \in \mathbb{N}, y_{1}, \ldots, y_{n} \in S_{2}, \beta_{1}, \gamma_{1}, \ldots, \beta_{n}, \gamma_{n} \in L$ such that

$$
\begin{aligned}
& \left(x, y_{1}, \beta_{1}\right), \ldots,\left(x, y_{n}, \beta_{n}\right) \in R,\left(y_{1}, z^{\prime}, \gamma_{1}\right), \ldots,\left(y_{n}, z^{\prime}, \gamma_{n}\right) \in Q, \\
& \beta_{1} * \gamma_{1} \vee \cdots \vee \beta_{n} * \gamma_{n} \geqslant \alpha^{\prime} .
\end{aligned}
$$

The simplest operation " $*$ " that obviously satisfies the above conditions is the lattice meet " $\wedge$ ". In this case for "*”" we use the denotation ";".

For an $L$-ambiguous representation $R$ we regard $R^{\smile}$ as a $\hat{L}$-ambiguous representation, and use " $\hat{*}$ " for the compositions of such representations.

Lemma 3 For all L-ambiguous representations $R \subset S_{1} \times S_{2} \times L, Q \subset S_{2} \times S_{3} \times L$ the inclusion $Q^{\smile *} R^{\smile} \subset(R, Q)^{\smile}$ holds.

Proof Let $(\hat{z}, \hat{x}, \alpha) \in Q^{{ }^{*},} R^{\smile}$. For each $\alpha^{\prime} \ll \alpha$ we can choose $\alpha^{\prime \prime}$ such that $\alpha^{\prime} \ll \alpha^{\prime \prime} \ll \alpha$, then for all $\hat{x}^{\prime} \ll \hat{x}$ there are $n \in \mathbb{N}, \hat{y}_{1}, \ldots, \hat{y}_{n} \in S_{2}, \gamma_{1}, \beta_{1}, \ldots, \gamma_{n}, \beta_{n} \in L$ such that

$$
\begin{aligned}
& \left(\hat{z}, \hat{y}_{1}, \gamma_{1}\right), \ldots,\left(\hat{z}, \hat{y}_{n}, \gamma_{n}\right) \in Q^{\smile},\left(\hat{y}_{1}, \hat{x}^{\prime}, \beta_{1}\right), \ldots,\left(\hat{y}_{n}, \hat{x}^{\prime}, \beta_{n}\right) \in R^{\smile}, \\
& \gamma_{1} \hat{*} \beta_{1} \vee \cdots \vee \gamma_{n} \hat{*} \beta_{n}=\beta_{1} * \gamma_{1} \vee \cdots \vee \beta_{n} * \gamma_{n} \geqslant \alpha^{\prime \prime} .
\end{aligned}
$$

For all $x \in S_{1}$ such that $x P_{1} \hat{x}^{\prime}=1$, and all $\beta_{1}^{\prime} \ll \beta_{1}, \ldots, \beta_{n}^{\prime} \ll \beta_{n}$ there are $y_{1}, \ldots, y_{n} \in S_{2}$ such that $\left(x, y_{1}, \beta_{1}^{\prime}\right), \ldots,\left(x, y_{n}, \beta_{n}^{\prime}\right) \in R, y_{1} P_{2} \hat{y}_{1}=\cdots=y_{n} P_{2} \hat{y}_{n}=1$. Analogously, for all $\gamma_{1}^{\prime} \ll \gamma_{1}, \ldots, \gamma_{n}^{\prime} \ll \gamma_{n}$ there are $z_{1}, \ldots, z_{n} \in S_{3}$ such that 
$\left(y_{1}, z_{1}, \gamma_{1}^{\prime}\right), \ldots,\left(y_{n}, z_{n}, \gamma_{n}^{\prime}\right) \in Q, z_{1} P_{3} \hat{z}=\cdots=z_{n} P_{3} \hat{z}=1$. Then the element $z=z_{1} \wedge \cdots \wedge z_{n}$ satisfies $z P_{3} \hat{z}=1$ and $\left(y_{1}, z, \gamma_{1}^{\prime}\right), \ldots,\left(y_{n}, z, \gamma_{n}^{\prime}\right) \in Q$ as well.

Obviously $\left(x, z, \beta_{1}^{\prime} * \gamma_{1}^{\prime} \vee \cdots \vee \beta_{n}^{\prime} * \gamma_{n}^{\prime}\right) \in R * Q$. Due to Scott continuity (i.e., lower semicontinuity) of $*$ and $\vee$, we can choose the above $\beta_{1}^{\prime}, \ldots, \beta_{n}^{\prime}, \gamma_{1}^{\prime}, \ldots, \gamma_{n}^{\prime}$ so that $\beta_{1}^{\prime} *$ $\gamma_{1}^{\prime} \vee \cdots \vee \beta_{n}^{\prime} * \gamma_{n}^{\prime} \geqslant \alpha^{\prime}$. Hence for all $\alpha^{\prime} \ll \alpha, \hat{x}^{\prime} \ll \hat{x}, x \in S_{1}, x P_{1} \hat{x}^{\prime}$ there is $z \in S_{3}$ such that $z P_{3} \hat{z}=1,\left(x, z, \alpha^{\prime}\right) \in R^{*} Q$, i.e., $(\hat{z}, \hat{x}, \alpha) \in(R, Q)^{\smile}$.

Mutatis mutandis we obtain an analogue of a statement for crisp ambiguous representations:

Corollary 2 Let L-ambiguous representations $R \subset S_{1} \times S_{2} \times L, Q \subset S_{2} \times S_{3} \times L$ be pseudoinvertible. Then $Q^{\smile{ }^{*}} R^{\smile}=\left(R^{*}, Q\right)^{\smile}$, and the composition $R *{ }^{*} Q$ is pseudo-invertible as well.

Proposition 10 Composition “,”, of the pseudo-invertible L-ambiguous representations is associative.

Proof is similar to the one for crisp representations and reduces to the observation that, for $L$-ambiguous representations $R \subset S_{1} \times S_{2} \times L, Q \subset S_{2} \times S_{3} \times L, S \subset S_{3} \times S_{4} \times L$, both statements $(x, t, \alpha) \in(R, Q), S$ and $(x, t, \alpha) \in R^{*},(Q, S)$ are equivalent to the existence, for all $\alpha^{\prime} \ll \alpha$ and $t^{\prime} \ll t$, of $n \in \mathbb{N}, y_{1}, \ldots, y_{n} \in S_{2}, z_{1}, \ldots, z_{n} \in S_{3}$, $\beta_{1}, \gamma_{1}, \delta_{1}, \ldots, \beta_{n}, \gamma_{n}, \delta_{n} \in L$ such that

$$
\begin{aligned}
& \left(x, y_{1}, \beta_{1}\right), \ldots,\left(x, y_{n}, \beta_{n}\right) \in R, \quad\left(y_{1}, z_{1}, \gamma_{1}\right), \ldots,\left(y_{n}, z_{n}, \gamma_{n}\right) \in Q, \\
& \left(z_{1}, t^{\prime}, \delta_{1}\right), \ldots,\left(z_{n}, t^{\prime}, \delta_{n}\right) \in S, \quad \beta_{1} * \gamma_{1} * \delta_{1} \vee \cdots \vee \beta_{n} * \gamma_{n} * \delta_{n} \geqslant \alpha^{\prime} .
\end{aligned}
$$

Hence we obtain a category:

Proposition 11 All continuous semilattices with bottom elements and all pseudo-invertible $L$-ambiguous representations form a category $\mathcal{S} \operatorname{em} \mathcal{P} \mathcal{R}_{L}^{*}$, which contains $\mathcal{S} \operatorname{em} \mathcal{P} \mathcal{R}$ as a subcategory.

The embedding $I_{L}^{*}: \mathcal{S}$ em $\mathcal{P} \mathcal{R} \rightarrow \mathcal{S}$ em $\mathcal{P} \mathcal{R}_{L}^{*}$ preserves the objects and turns each crisp ambiguous representation $R \subset S_{1} \times S_{2}$ into an $L$-ambiguous one as follows:

$$
I_{L}^{*} R=\left\{(x, y, \alpha) \subset S_{1} \times S_{2} \times L \mid(x, y) \in R \text { or } \alpha=0\right\} .
$$

Clearly there is also the embedding $I_{L}^{\hat{*}}: \mathcal{S e m} \mathcal{P} \mathcal{R} \rightarrow \mathcal{S e m} \mathcal{P} \mathcal{R}_{L}^{\hat{*}}$ into the category built upon the "swapped" operation " $\hat{*}$ ".

We denote an arrow $R$ from $S_{1}$ to $S_{2}$ with $R: S_{1} \Rightarrow^{*} S_{2}$ in $\mathcal{S e m} \mathcal{P} \mathcal{R}_{L}^{*}$ and with $R: S_{1} \Rightarrow^{*} S_{2}$ in $\mathcal{S e m} \mathcal{P} \mathcal{R}_{L}^{*}$. The composition of $R$ and $Q$ is denoted by $R{ }^{*}, Q$ (in direct order) or $Q \circledast R$ (in reverse order) in $\mathcal{S e m} \mathcal{P} \mathcal{R}_{L}^{*}$, and by $R{ }^{*} Q$ or $Q \hat{\circledast} R$ respectively in $\mathcal{S e m} \mathcal{P} \mathcal{R}_{L}^{*}$.

Proposition 12 The self-duality $(-)^{\smile}: \mathcal{S}$ em $\mathcal{P} \mathcal{R} \rightarrow \mathcal{S e m} \mathcal{P} \mathcal{R}$ extends to contravariant functors $(-)^{\smile}: \mathcal{S e m}_{\mathcal{P}} \mathcal{R}_{L}^{*} \rightarrow \mathcal{S e m} \mathcal{P} \mathcal{R}_{L}^{\hat{*}}$ and $(-)^{\smile}: \mathcal{S e m} \mathcal{P} \mathcal{R}_{L}^{*} \rightarrow \mathcal{S e m} \mathcal{P} \mathcal{R}_{L}^{*}$. Both pairwise compositions of these functors are isomorphic to the identity functors.

Hence $(-)^{\smile}$ is a "contravariant equivalence" between $\mathcal{S e m} \mathcal{P} \mathcal{R}_{L}^{*}$ and $\mathcal{S}$ em $\mathcal{P} \mathcal{R}_{L}^{\hat{*}}$. 


\section{Ambiguous Representations as Affine Operators and Predicate Transformers}

Observe that compositions of (crisp or $L$-fuzzy) ambiguous representations were calculated only for their "relational" forms, but not for them given as isotone mappings. Interpretation of "slicewise defined" pseudo-inverses to $L$-ambiguous representations is also unclear. To fill this gap, we briefly present results of [14] and show the connection with the developed theory.

Recall that we treat $*$ as a (possibly noncommutative) conjunction in an $L$-valued fuzzy logic, and $\oplus=\vee$ will be a disjunction. The Boolean case is obtained for $L=\{0,1\}$ and $*=\wedge$.

A (left idempotent) $(L, \oplus, *)$-semimodule [1] (or $L$-semimodule for short if the operations $\oplus$ and $*$ are clearly understood) is a set $X$ with operations $\bar{\oplus}: X \times X \rightarrow X$ and $\bar{*}: L \times X \rightarrow X$ such that for all $x, y, z \in X, \alpha, \beta \in L$ :

(1) $x \bar{\oplus} y=y \bar{\oplus} x$

(2) $(x \bar{\oplus} y) \bar{\oplus} z=x \bar{\oplus}(y \bar{\oplus} z)$;

(3) there is an (obviously unique) element $\overline{0} \in X$ such that $x \bar{\oplus} \overline{0}=x$ for all $x$;

(4) $\alpha \bar{*}(x \bar{\oplus} y)=(\alpha \bar{*} x) \bar{\oplus}(\alpha \bar{*} y),(\alpha \oplus \beta) \bar{*} x=(\alpha \bar{*} x) \bar{\oplus}(\beta \bar{*} x)$;

(5) $\quad(\alpha * \beta) \bar{*} x=\alpha \bar{*}(\beta \bar{*} x)$;

(6) $1 \bar{*} x=x$; and

(7) $0 \bar{*} x=\overline{0}$

Observe that these axioms imply that $(X, \bar{\oplus})$ is an upper semilattice with a bottom element $\overline{0}$, the order is defined as $x \leqslant y \Longleftrightarrow x \bar{\oplus} y=y$, and $\alpha \bar{*} \overline{0}=\overline{0}$ for all $\alpha \in L$. The operation $\bar{*}$ is isotone in both variables.

Hence an $(L, \oplus, *)$-semimodule is an analogue of a vector space. Similarly, analogues exist for linear and affine mappings. A mapping $f: X \rightarrow Y$ between $(L, \oplus, *)$ semimodules is called linear if, for all $x_{1}, \ldots, x_{n} \in X$ and $\alpha_{1}, \ldots, \alpha_{n} \in L$, the equality

$$
f\left(\alpha_{1} \bar{*} x_{1} \bar{\oplus} \ldots \bar{\oplus} \alpha_{n} \bar{*} x_{n}\right)=\alpha_{1} \bar{*} f\left(x_{1}\right) \bar{\oplus} \ldots \bar{\oplus} \alpha_{n} \bar{*} f\left(x_{n}\right)
$$

is valid. If the latter equality is ensured only whenever $\alpha_{1} \oplus \ldots \oplus \alpha_{n}=1$, then $f$ is called affine. Observe that an affine mapping $f$ preserves joins, i.e. $f\left(x_{1} \bar{\oplus} x_{2}\right)=f\left(x_{1}\right) \bar{\oplus} f\left(x_{2}\right)$ for all $x_{1}, x_{2} \in X$. An affine mapping is linear if and only if it preserves the least element.

We call a triple $(X, \bar{\oplus}, \bar{*})$ a continuous $(L, \oplus, *)$-semimodule if $(X, \bar{\oplus}, \bar{*})$ is an $(L, \oplus, *)$-semimodule such that $(X, \bar{\oplus})$ is a domain (therefore is a complete lattice), and * is infinitely distributive w.r.t. supremum in the both variables (hence is Scott continuous). Observe that such $(X, \bar{\oplus})$ has a least element, a greatest element, and suprema for all subsets, therefore is a continuous lattice. If the poset $(X, \bar{\oplus})$ is a completely distributive lattice, then we call $(X, \bar{\oplus}, \bar{*})$ a completely distributive $(L, \oplus, *)$-semimodule.

It was proved [14] that, for a domain $D$ with a bottom element, $\left(M_{[L]} D, \bar{\oplus}, \bar{\circledast}\right)$ is a completely distributive $(L, \oplus, *)$-semimodule with the operations

$$
\begin{gathered}
\left(m \bar{\oplus} m^{\prime}\right)(b)=m(b) \oplus m^{\prime}(b), \quad b \in D, \\
(\alpha \odot m)(b)= \begin{cases}(\alpha \bar{\oplus} m)(b)=\inf \{\alpha * m(a) \mid a \in D, a \ll b\}, & b \neq 0 ; \quad b=0, \\
1, & b \in L, a \in D .\end{cases}
\end{gathered}
$$

For an element $d_{0} \in D$, we denote by $\eta_{[L]} D\left(d_{0}\right)$ the function $D \rightarrow L$ that sends each $d \in$ $D$ to 1 if $d \leqslant d_{0}$ and to 0 otherwise. It is easy to see that $\eta_{[L]} D\left(d_{0}\right) \in M_{[L]} D$, and $\eta_{[L]} D(0)$ is a least element of $M_{[L]} D$. It follows from [16, Lemma 1.1] that the mapping $\eta_{[L]} D: D \rightarrow$ $M_{[L]} D$ is Scott continuous and lower continuous. Moreover, if $D$ is a continuous semilattice 
with zero, then $\eta_{[L]} D$ is a zero-preserving semilattice morphism, hence we consider $D$ as a subset of $M_{[L]} D$.

Proposition 13 ([14], 1.3) For each Scott continuous mapping $\varphi: D \rightarrow K$ from a domain with a bottom element to a continuous L-semimodule there is a unique Scott continuous affine extension $\Phi: M_{[L]} D \rightarrow K$. It is linear if and only if $\varphi$ preserves the bottom element.

The required extension is determined by the formula

$$
\Phi(m)=\sup \{m(d) \bar{*} \varphi(d) \mid d \in D\}
$$

for all $m \in M_{[L]} D$.

This means that $M_{[L]} D$ is a free object in the category $(L, \oplus, *)-\mathcal{C} \mathcal{S} \mathcal{M} \mathrm{dod}_{\uparrow}$ of continuous $L$-semimodules and Scott continuous linear mappings over an object $D$ of the category $\mathcal{D o m}_{\uparrow}$ of domains with bottom elements and their Scott continuous bottom-preserving mappings.

An immediate corollary (cf. [14], proof of Proposition 2.3) is that, for domains $D_{1}, D_{2}$ with bottom elements, each Scott continuous linear mapping $\Phi: M_{[L]} D_{1} \rightarrow M_{[L]} D_{2}$ is uniquely determined with its restriction $\varphi: D_{1} \rightarrow M_{[L]} D_{2}$, i.e., with the composition $\varphi=\Phi \circ \eta_{[L] D_{1}}$. Recall that, for continuous semilattices $S_{1}, S_{2}$ with zeros, a Scott continuous bottom-preserving mapping $S_{1} \rightarrow M_{[L]} S_{2}$ is precisely a pseudo-invertible $L$-ambiguous representation.

Proposition 14 Let, for pseudo-invertible L-ambiguous representations $\varphi: S_{1} \Rightarrow{ }^{*} S_{2}$ and $\psi: S_{2} \Rightarrow^{*} S_{3}, \Phi: M_{[L]} S_{1} \rightarrow M_{[L]} S_{2}$ and $\Psi: M_{[L]} S_{2} \rightarrow M_{[L]} S_{3}$ be the Scott continuous linear extensions. Then the composition $\psi \circledast \varphi$ in $\mathcal{S e m}_{\mathcal{P}} \mathcal{R}_{L}^{*}$ is the restriction of $\Psi \circ \Phi: M_{[L]} S_{1} \rightarrow M_{[L]} S_{3}$.

Proof It is sufficient to verify that $\Psi \circ \varphi: S_{1} \rightarrow M_{[L]} S_{3}$ coincides with $\psi \circledast \varphi$. For all $x \in S_{1}$

$$
\Psi \circ \varphi(x)=\sup \left\{\varphi(x)(y) \circledast \bar{\circledast} \psi(y) \mid y \in S_{2}\right\},
$$

hence for all $z \in S_{3} \backslash\left\{0_{3}\right\}$

$$
\begin{aligned}
(\Psi \circ \varphi(x))(z) & =\inf \left\{\sup \left\{(\varphi(x)(y) \odot \psi(y))\left(z^{\prime}\right) \mid y \in S_{2}\right\} \mid z^{\prime} \ll z\right\} \\
& =\inf \left\{\sup \left\{\inf \left\{\varphi(x)(y) * \psi(y)\left(z^{\prime \prime}\right) \mid z^{\prime \prime} \ll z^{\prime}\right\} \mid y \in S_{2}\right\} \mid z^{\prime} \ll z\right\} .
\end{aligned}
$$

Observe that $\inf \left\{\varphi(x)(y) * \psi(y)\left(z^{\prime \prime}\right) \mid z^{\prime \prime} \ll z^{\prime}\right\} \geqslant \varphi(x)(y) * \psi(y)\left(z^{\prime}\right)$, hence

$$
\begin{aligned}
& \inf \left\{\sup \left\{\inf \left\{\varphi(x)(y) * \psi(y)\left(z^{\prime \prime}\right) \mid z^{\prime \prime} \ll z^{\prime}\right\} \mid y \in S_{2}\right\} \mid z^{\prime} \ll z\right\} \\
\geqslant & \inf \left\{\sup \left\{\varphi(x)(y) * \psi(y)\left(z^{\prime}\right) \mid y \in S_{2}\right\} \mid z^{\prime} \ll z\right\} \\
= & \inf \left\{\sup \left\{\varphi(x)(y) * \psi(y)\left(z^{\prime \prime}\right) \mid y \in S_{2}\right\} \mid z^{\prime \prime} \ll z\right\}
\end{aligned}
$$

(in the last line we just rename $z^{\prime}$ with $z^{\prime \prime}$.)

On the other hand, for all $z^{\prime \prime} \ll z$ in a continuous semilattice there is $z^{\prime}$ such that $z^{\prime \prime} \ll$ $z^{\prime} \ll z$, for which

$$
\varphi(x)(y) * \psi(y)\left(z^{\prime \prime}\right) \geqslant \inf \left\{\varphi(x)(y) * \psi(y)\left(z^{\prime \prime}\right) \mid z^{\prime \prime} \ll z^{\prime}\right\},
$$

hence

$$
\begin{aligned}
& \inf \left\{\sup \left\{\varphi(x)(y) * \psi(y)\left(z^{\prime \prime}\right) \mid y \in S_{2}\right\} \mid z^{\prime \prime} \ll z\right\} \\
\geqslant & \inf \left\{\sup \left\{\inf \left\{\varphi(x)(y) * \psi(y)\left(z^{\prime \prime}\right) \mid z^{\prime \prime} \ll z^{\prime}\right\} \mid y \in S_{2}\right\} \mid z^{\prime} \ll z\right\} .
\end{aligned}
$$


Thus we obtain for all $z \in S_{3} \backslash\left\{0_{3}\right\}$

$$
(\Psi \circ \varphi(x))(z)=\inf \left\{\sup \left\{\varphi(x)(y) * \psi(y)\left(z^{\prime}\right) \mid y \in S_{2}\right\} \mid z^{\prime} \ll z\right\},
$$

therefore $(\Psi \circ \varphi(x))(z) \geqslant \alpha \in L$ if and only if for all $z^{\prime} \ll z$ and $\alpha^{\prime} \ll \alpha$ we have $\alpha^{\prime} \ll \sup \left\{\varphi(x)(y) * \psi(y)\left(z^{\prime}\right) \mid y \in S_{2}\right\}$. As the least upper bound of a set $A$ in $L$ is the limit of the directed net of the least upper bounds of the finite subsets of $A$, the latter condition is equivalent to existence, for all $\alpha^{\prime} \ll \alpha$ and $z^{\prime} \ll z$, of $y_{1}, y_{2}, \ldots, y_{n} \in S_{2}$ such that

$$
\varphi(x)\left(y_{1}\right) * \psi\left(y_{1}\right)\left(z^{\prime}\right) \vee \varphi(x)\left(y_{2}\right) * \psi\left(y_{2}\right)\left(z^{\prime}\right) \vee \cdots \vee \varphi(x)\left(y_{n}\right) * \psi\left(y_{n}\right)\left(z^{\prime}\right) \geqslant \alpha^{\prime} .
$$

Recall that $(x, z, \alpha) \in \psi థ \bar{\circledast} \varphi$ if and only if for all $z^{\prime} \ll z, \alpha^{\prime} \ll \alpha$ there are $n \in \mathbb{N}$, $y_{1}, \ldots, y_{n} \in S_{2}, \beta_{1}, \gamma_{1}, \ldots, \beta_{n}, \gamma_{n} \in L$ such that

$$
\begin{aligned}
& \left(x, y_{1}, \beta_{1}\right), \ldots,\left(x, y_{n}, \beta_{n}\right) \in \varphi,\left(y_{1}, z^{\prime}, \gamma_{1}\right), \ldots,\left(y_{n}, z^{\prime}, \gamma_{n}\right) \in \psi, \\
& \beta_{1} * \gamma_{1} \vee \cdots \vee \beta_{n} * \gamma_{n} \geqslant \alpha^{\prime} .
\end{aligned}
$$

Clearly $(\Psi \circ \varphi(x))(z) \geqslant \alpha$ if and only if $(x, z, \alpha) \in \psi \bar{\circledast} \varphi$, and the proof is complete.

Thus we arrive at:

Proposition 15 The correspondence that assigns $M_{[L]} S$ to each continuous semilattice $S$ and the unique Scott continuous linear extension $M_{[L]} S_{1} \rightarrow M_{[L]} S_{2}$ to each Scott continuous bottom-preserving mapping $S_{1} \rightarrow M_{[L]} S_{2}{ }^{1}$ is a functor that embeds the category $\mathcal{S e m} \mathcal{P} \mathcal{R}_{L}^{*}$ into the category $(L, \oplus, *)-\mathcal{C S} \mathcal{S} \operatorname{dod}_{\uparrow}$ of continuous L-semimodules as a full subcategory.

In fact the latter subcategory is the Kleisli category for the respective monad [10], but we will not go into detail here. Now it is obvious how to compose $L$-ambiguous representation in "functional" form: first extend them to linear mappings of free semimodules, then compose in $(L, \oplus, *)-\mathcal{C S} \mathcal{M o d}_{\uparrow}$, and, finally, restrict back to semigroups.

Now it is time to present practical sense of the constructed extensions, following [16]. For a domain of computation $D$, we treat each mapping $m: D \rightarrow L$ as "it is known that, for each $d \in D$, its truth value is (or can be) at least $m(d)$ ". Similarly, an arbitrary mapping $\varphi: D \rightarrow \underline{M}_{[L]} D^{\prime}$ is interpreted as "if $a \in D$ is true, then the truth value of each $b \in D^{\prime}$ is at least $\varphi(a)(b)$ ". Note that $\varphi(a)(b)$ is implicitly considered as a "conditional" truth value, i.e., if $a$ is "partially true" at a degree $\geqslant \alpha$, then $b$ is true at least at a degree $\alpha * \varphi(a)(b)$.

Hence, such a $\varphi$ is an $L$-fuzzy state transformer. For a given $\varphi$, we say that $m: D \rightarrow L$ is a precondition and $m^{\prime}: D^{\prime} \rightarrow L$ is a postcondition for each other w.r.t. $\varphi$, if, for all $a \in D$ and $b \in D^{\prime}$, the "guaranteed" truth value $m^{\prime}(b)$ is greater or equal to $m(a) * \varphi(a)(b)$, i.e., to the result of modus ponens.

Obviously, for an antitone function $m: D \rightarrow L$, its strongest (least) postcondition $\Phi(m)$ in $\underline{M}_{[L]} D^{\prime}$ is determined by the equality

$$
\Phi(m)(b)=\inf \left\{\sup \left\{m(a) * \varphi(a)\left(b^{\prime}\right) \mid a \in D\right\} \mid b^{\prime} \in D^{\prime}, b^{\prime} \ll b\right\}, b \in D^{\prime} .
$$

The same formula is valid also for normalized predicates, and it is easy to observe that the strongest postcondition predicate transformer $\Phi$ is exactly the unique linear Scott continuous extension of the state transformer $\varphi$.

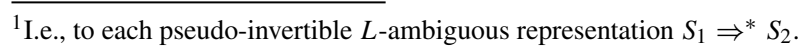




\section{Pseudo-Inverses as Hermitian Conjugates}

To clarify what the pseudo-inverse to an ambiguous representation is, we use a "scalar-like" product of monotonic predicates, which was introduced in [14].

Let $S$ be a continuous semilattice with bottom elements, and $P: S \times S^{\prime} \rightarrow\{0,1\}$ be a separating compatibility. We regard $M_{[L]} S$ as a continuous $L$-semimodule and $M_{[L]} \hat{S}$ as a continuous $\hat{L}$-semimodule, where $\hat{L}$ is $L$ with the "swapped" multiplication.

For monotonic predicates $m: S \rightarrow L, m^{\prime}: S^{\prime} \rightarrow L$ let

$$
\left(m, m^{\prime}\right)_{P}^{*}=m \cdot m^{\prime}=\sup \left\{m(d) * P\left(d, d^{\prime}\right) * m^{\prime}\left(d^{\prime}\right) \mid d \in S, d^{\prime} \in S^{\prime}\right\} .
$$

We use the second notation if $P$ and $*$ are easily guessed. It was proved that the introduced multiplication is infinitely distributive w.r.t. joins and uniform in the both arguments. Moreover, by Proposition $2.2[14] M_{[L]} S$ and $M_{[L]} S^{\prime}$, together with the multiplication $(-,-)_{P}^{*}: M_{[L]} S \times M_{[L]} S^{\prime} \rightarrow L$, constitute a dual pair [6], i.e., the multiplication separates elements, both of $M_{[L]} S$ and of $M_{[L]} S^{\prime}$, in the obvious sense.

If to incompatible $s \in S, s^{\prime} \in S^{\prime}$ monotonic predicates $m$ and $m^{\prime}$ assign truth values $m(s)$ and $m^{\prime}\left(s^{\prime}\right)$ with non-zero conjunction $m(s) * m^{\prime}\left(s^{\prime}\right)$, then the predicates are also incompatible to this extent. The product $\left(m, m^{\prime}\right)_{P}^{*}$ is precisely the supremum of such "incompatibilities" $m(s) * m^{\prime}\left(s^{\prime}\right)$, for $s P s^{\prime}=1$, hence it can be regarded as a measure of total incompatibility of $m$ and $m^{\prime}$.

The following notion was also introduced in [14]. Assume that $K_{1}, K_{2}$ are $L$ semimodules, $K_{1}^{\prime}, K_{2}^{\prime}$ are $\hat{L}$-semimodules, multiplications $\cdot: K_{1} \times K_{1}^{\prime} \rightarrow L$ and $\cdot: K_{2} \times$ $K_{2}^{\prime} \rightarrow L$ are such that $K_{1}, K_{1}^{\prime}$ and $K_{2}, K_{2}^{\prime}$ are dual pairs, and $A: K_{1} \rightarrow K_{2}$ is a linear mapping. It is natural to call a linear mapping $A^{\prime}: K_{2}^{\prime} \rightarrow K_{1}^{\prime}$ the (Hermitian) conjugate to $A$ if $A a \cdot a^{\prime}=a \cdot A^{\prime} a^{\prime}$ whenever $a \in K_{1}, a \in K_{2}^{\prime}$. The separation property implies that, if a conjugate for a given $A$ exists, it is unique. Hence we write $A^{\prime}=A^{*}$ in this case, and obviously $A^{* *}=A$. It is also immediate that, for the composition $A \circ B$ of linear mappings with conjugates $A^{*}$ and $B^{*}$, respectively, a conjugate exists and is equal to $B^{*} \circ A^{*}$.

Proposition 16 ([14], 2.3) Let $S_{1}, S_{2}$ be continuous semilattices with bottom elements. For each Scott continuous linear mapping $\Phi: M_{[L]} S_{1} \rightarrow M_{[L]} S_{2}$ there is a Scott continuous conjugate $\Phi^{\prime}: M_{[L]} S_{2}^{\wedge} \rightarrow M_{[L]} S_{1}^{\wedge}$.

Recall that $\Phi$ and $\Phi^{\prime}$ are the unique Scott continuous linear extensions of $L$-ambiguous pseudo-invertible representations $\varphi: S_{1} \rightarrow M_{[L]} S_{2}$ and $\varphi^{\prime}: S_{2}^{\wedge} \rightarrow M_{[L]} S_{1}^{\wedge}$. Given $\varphi$, one can calculate $\varphi^{\prime}$ as follows:

$$
\varphi^{\prime}(\hat{y})(\hat{x})=\inf \{\sup \{\varphi(x)(y) \mid y \in \hat{y}\} \mid x \in \hat{x}\},
$$

or, in terms of compatibilities:

$$
\varphi^{\prime}(\hat{y})(\hat{x})=\inf \left\{\sup \left\{\varphi(x)(y) \mid y \in S_{2}, y P_{2} \hat{y}=1\right\} \mid x \in S_{1}, x P_{1} \hat{x}=1\right\} .
$$

Hence $\varphi^{\prime}(\hat{y})(\hat{x}) \geqslant \alpha \neq 0$ if and only if for all $x \in S_{1}$ such that $x P_{1} \hat{x}=1$ and for all $\beta \ll \alpha$ there are $y_{1}, y_{2}, \ldots, y_{n} \in S_{2}$ such that $y_{1} P_{2} \hat{y}=y_{2} P_{2} \hat{y}=\cdots=y_{n} P_{2} \hat{y}=1$ and

$$
\varphi(x)\left(y_{1}\right) \vee \varphi(x)\left(y_{2}\right) \vee \cdots \vee \varphi(x)\left(y_{n}\right) \geqslant \beta .
$$

Then for $y=y_{1} \vee y_{2} \vee \cdots \vee y_{n}$ we have $y P_{2} \hat{y}=1$ and $\varphi(x)(y) \geqslant \beta$. 
Thus $\varphi^{\prime}(\hat{y})(\hat{x}) \geqslant \alpha$ if and only if for all $x \in S_{1}$ such that $x P_{1} \hat{x}=1$ and for all $\beta \ll \alpha$ there is $y \in S_{2}$ such that $y P_{2} \hat{y}=1$ and $\varphi(x)(y \geqslant \beta$. This exactly means that $(\hat{y}, \hat{x}, \alpha) \in$ $\varphi^{\smile}$, hence $\varphi^{\prime}=\varphi^{\smile}$. We arrive at a conclusion:

Proposition 17 If a mapping $\Phi: M_{[L]} S_{1} \rightarrow M_{[L]} S_{2}$ is the Scott continuous linear extension of a pseudo-invertible L-ambiguous representation $\varphi: S_{1} \Rightarrow{ }^{*} S_{2}$, then the conjugate mapping $\Phi^{\prime}: M_{[L]} S_{2}^{\wedge} \rightarrow M_{[L]} S_{1}^{\wedge}$ is the Scott continuous linear extension of the pseudo-inverse L-ambiguous representation $\varphi^{\smile}: S_{2}^{\wedge} \Rightarrow^{*} \hat{S}_{1}^{\wedge}$.

\section{Concluding Remarks and Future Work}

What we proposed is just a sketch of application of crisp and $L$-fuzzy ambiguous representations of continuous semillattices. It is easy to note that properties of $\mathcal{S e m} \mathcal{P} \mathcal{R}$ are similar to ones of dialogue category, with $\{0,1\}$ being a tensorial pole, and the pair $\operatorname{Sem} \mathcal{P} \mathcal{R}_{L}^{*}$, $\mathcal{S}$ em $\mathcal{P} \mathcal{R}_{L}^{*}$ looks like a dialogue chirality [11]. Than taking pseudo-inverse could be tensorial negation. Unfortunately, it is not the case, e.g., $\{0,1\}$ is not exponentiable in $\mathcal{S}$ em $\mathcal{P} \mathcal{R}$, similarly verification fails for $\mathcal{S} \operatorname{em} \mathcal{P} \mathcal{R}_{L}^{*}$ and $\mathcal{S}$ em $\mathcal{P} \mathcal{R}_{L}^{*}$. Nevertheless, the similarity is not incidental, and we plan to continue this research to model games in normal and extended form, winning strategies etc. We are also going to show why completely distributive lattices arise in games with imperfect information.

Authors also express deepest gratitude to Prof. Michael Zarichnyi for valuable ideas.

Open Access This article is distributed under the terms of the Creative Commons Attribution 4.0 International License (http://creativecommons.org/licenses/by/4.0/), which permits unrestricted use, distribution, and reproduction in any medium, provided you give appropriate credit to the original author(s) and the source, provide a link to the Creative Commons license, and indicate if changes were made.

\section{References}

1. Akian, M.: Densities of invariant measures and large deviations. Trans. Amer. Math. Soc. 351(11), 45154543 (1999)

2. Bergmann, M.: Introduction to many-valued and fuzzy logic: Semantics, algebras, and derivation systems. Cambridge University Press, New York (2008)

3. Edalat, A.: Domains for computation in mathematics, physics and exact real arithmetic. Bull. Symb. Logic 3(4), 401-452 (1997)

4. Erné, M.: Z-distributive function spaces preprint (1998)

5. Gierz, G., Hofmann, K.H., Keimel, K., Lawson, J.D., Mislove, M., Scott, D.S., Lattices, C.ontinuous.: Domains encyclopedia of mathematics and its applications, vol. 93. Cambridge University Press, New York (2003)

6. Cohen, G., Gaubert, S., Quadrat J.-P.: Duality and separation theorems in idempotent semimodules. arXiv:0212294v2 [math.FA], 29 (2003)

7. Hájek, P.: Fuzzy logics with noncommutative conjuctions. J. Logic Computation 13(4), 469-479 (2003)

8. Heckmann, R., Huth, M.: A duality theory for quantitative semantics. In: Proceedings of the 11th International Workshop on Computer Science Logic, vol. 1414 of Lecture Notes in Computer Science. Springer Verlag, 255-274 (1998)

9. Johnstone, P.T.: Stone spaces Cambridge studies in advanced mathematics, vol. 3. Cambridge University Press, New York (1983)

10. Mac Lane, S. Categories for the Working Mathematician, 2nd edn. Springer, New York (1998)

11. Melliès, P.-A.: Dialogue categories and chiralities. Publ. Res. Inst. Math. Sci. 52(4), 359-412 (2016)

12. Nykyforchyn, O.R.: Capacities with values in compact Hausdorff lattices. Appl. Categ. Struct. 15(3), 243-257 (2008) 
13. Nykyforchyn, O., Mykytsey, O.: Conjugate measures on semilattices. Visnyk Lviv Univ. 72, 221-231 (2010)

14. Nykyforchyn, O., Mykytsey, O.: L-idempotent linear operators between predicate semimodules, dual pairs and conjugate operator. Mathematical Bulletin of the Shevchenko Scientific Society 8, 299-314 (2011)

15. Nykyforchyn, O., Repovš, D.: Ambiguous representations as fuzzy relations between sets. Fuzzy Set. Syst. 173, 25-44 (2011)

16. Nykyforchyn, O.R., Repovš, D.: $L$-fuzzy strongest postcondition predicate transformers as $L$-idempotent linear or affine operators between semimodules of monotonic predicates. Fuzzy Set. Syst. 208, 67-78 (2012)

17. Scott, D.S.Nielsen, M., Schmidt, E.M. (eds.): Domains for denotational semantics. Springer, Berlin (1982)

Publisher's Note Springer Nature remains neutral with regard to jurisdictional claims in published maps and institutional affiliations. 